\title{
ELECTRON SPECTRUM AND DIELECTRIC SUSCEPTIBILITY OF THE HUBBARD MODEL WITH LOCAL LATTICE ANHARMONICITY
}

\author{
I.V. Stasyuk and A.M. Shvaika \\ Institute for Condensed Matter Physics, Ukrainian Academy of Sciences \\ Svientsitsky St. 1, Lviv 290011, Ukraine
}

The investigation of single-electron spectrum of the Hubbard model with local lattice anharmonicity is performed. The influence of the interaction with the vibrational subsystem on the effective exchange constant is considered. The static transverse dielectric susceptibility of the model is calculated. Analysis of the possible dielectric anomalies is performed on this basis.

PACS numbers: 74.20.Mn, 71.45.Gm

1. The model with local anharmonicity (Müller model) [1] involving Hubbard-type electron correlations as well as interaction between electrons and vibrational modes which can be represented in terms of pseudospin variables, is one of the used in the theory of high- $T_{\mathrm{c}}$ superconductivity. Vibrations of apex oxygen $\mathrm{O}_{\mathrm{IV}}$ possess anharmonicity in the case of YBaCuO-type systems. Their possible importance for charge transfer to or from the $\mathrm{CuO}$ layers, superconducting pairing of electrons and connection with observed high values of dielectric permittivity $\varepsilon_{z} \dot{z}$ was mentioned in several papers [2,3].

2. Hamiltonian of the model [1] has the following form:

$$
\begin{aligned}
& H=H_{0}+\sum_{i j \sigma} t_{i j} a_{i \sigma}^{\dagger} a_{j \sigma}, \\
& H_{0}=\sum_{i}\left(U n_{i \uparrow} n_{i \downarrow}+\left(g S_{i}^{z}-\mu\right) n_{i}-\Omega S_{i}^{x}-h S_{i}^{z}\right) .
\end{aligned}
$$

Here, $n_{i}=\sum_{\sigma} n_{i \sigma} ; n_{i \sigma}=a_{i \sigma}^{\dagger} a_{i \sigma}$ is the electron number operator at site $i$ with the spin $\sigma ; g n_{i} S_{i}^{z}$ describes interaction between electrons and anharmonic vibrations. The last two terms in $H_{0}$ describe tunnelling splitting of the vibrational mode and the asymmetry of the anharmonic potential; $t_{i j}$ is the electron transfer integral.

The Hamiltonian $H_{0}$ can be reduced to the diagonal form using the transformation $|R\rangle=\alpha_{R r}|r\rangle$ [4], where $|R\rangle=\left|n_{i \uparrow}, n_{i \downarrow}, S_{i}^{z}\right\rangle$ is the single-site basis of states. In the Hubbard operators representation $H_{0}=\sum_{r} \varepsilon_{r} X_{i}^{r r}$, where

$$
\varepsilon_{r, \tilde{r}}=U \delta_{r, 2}-n_{r} \mu \pm \frac{1}{2} \sqrt{\left(n_{r} g-h\right)^{2}+\Omega^{2}}
$$


Here $a_{i \sigma}^{\dagger}=\sum_{m, n} A_{m n}^{\sigma} X_{i}^{m n}, X^{m n}=|m\rangle\langle n| ; n_{1}=0, n_{2}=2, n_{3}=n_{4}=1\left(n_{r}=n_{\tilde{r}}\right)$ and states $|1\rangle,|2\rangle,|3\rangle,|4\rangle$ correspond to the hole, pair and single-electron states with $\sigma=\downarrow$, $\uparrow$, respectively (the states with $S_{i}^{z}=-\frac{1}{2}$ are marked by ).

3 . The problem of electron energy spectrum and electron statistics is solved using the method of two-time Green functions constructed of $X$-operators with the decoupling corresponding to Hubbard-I approximation (under the conditions $t \ll U, t \ll g)$. We obtain in the independent subband approximation $\lambda_{m n}(q)=$ $\varepsilon_{m}-\varepsilon_{n}+t_{q}\left(A_{m n}^{\sigma}\right)^{2}\left\langle X^{m m}+X^{n n}\right\rangle$. The four lower and four higher bands correspond to the hole and pair motions, respectively (see Fig. 1). This splitting is caused by interaction between Hubbard levels with the pseudospin subsystem.

There are double occupied sites below half-filling $(n=1-\delta)$ and empty sites above half-filling $(n=1+\delta)$ due to the interband interaction

$$
\left.\left\langle X^{\tilde{2} \tilde{2}}\right\rangle\right|_{n=1-\delta} \approx\left(\frac{t}{U_{\mathrm{eff}}}\right)^{2}\left(A_{41}^{\sigma}\right)^{4},\left.\quad\left\langle X^{11}\right\rangle\right|_{n=1+\delta} \approx\left(\frac{t}{U_{\mathrm{eff}}}\right)^{2}\left(A_{23}^{\sigma}\right)^{4}
$$

$\left(U_{\text {eff }}=\left(\varepsilon_{\tilde{2}}-\varepsilon_{\overline{3}}\right)-\left(\varepsilon_{\tilde{4}}-\varepsilon_{\tilde{1}}\right) \leq U\right)$. The electron-hole symmetry is broken and there is a possibility for the hole conductivity at half-filling.

Similarly to the standard transition from the Hubbard model to the $t-J$ one, the effective Hamiltonian is constructed with the help of the canonical transformation. It contains interaction of the form

$$
\sum_{i j \alpha \beta} \sum_{i j}^{\alpha \beta} P_{i}^{\alpha} P_{i}^{\beta}\left(\sigma \cdot \sigma-\frac{1}{4} n_{i} n_{j}\right),
$$

where the effective exchange constant depends on the state of vibrational subsystem $\left(P_{i}^{ \pm}=\frac{1}{2} \pm S_{i}^{z} ; P_{i}^{\uparrow, \downarrow}=S_{i}^{+,-}\right)$. In equilibrium state at low temperatures $\left\langle S_{i}^{z}\right\rangle \approx-\frac{1}{2}$ and $J_{i j}^{-}$(which is antiferromagnetic for $U_{\text {eff }}>0$ ) produces the main contribution in the total exchange. In nonequilibrium state (e.g. in the frozen disorder case) the number of sites with $S_{i}^{z}=+\frac{1}{2}$ increases and the components $J_{i j}^{+-}$and $J_{i j}^{++}$(which can be ferromagnetic for certain range of parameters values) give the contribution. This can correspond to the frustration of the exchange interaction discussed in some papers.

4. In calculations of the transverse dielectric susceptibility (corresponding to the component $\varepsilon_{z z}$ for $\mathrm{YBaCuO}$-structures) we start from the relation $\chi_{\perp}(q, \omega)=$ $\left\langle P_{\boldsymbol{q}}(\omega) P_{-\boldsymbol{q}}(\omega)\right\rangle^{c}$ containing the Fourier transform of the Matsubara temperature Green function constructed of operators $P_{i}=d_{\mathrm{S}} S_{i}^{z}+d_{\mathrm{e}} n_{i}$. Here, $d_{\mathrm{S}}$ is the dipole momentum connected with the pseudospin flipping; $d_{\mathrm{e}}$ is the electron component of the dipole momentum corresponding to charge transfer from/to the $\mathrm{CuO}$ layers. The calculation of correlators $\left\langle S^{z} S^{z}\right\rangle^{c},\langle n n\rangle^{c}$ etc. is performed with the use of Wick's theorem and corresponding diagrammatic technique. The operator $H_{0}$ is taken as Hamiltonian of zero approximation. Effective interaction between pseudospins via electron subsystem is taken into account using ladder approximation for the summation of loop diagrams and the electron propagators are taken in Hubbard-I approximation (this corresponds to the generalized random phase approximation [5]).

Dielectric susceptibility was found for two regimes: $\mu=$ const and $n=$ const. The dependencies of susceptibility $\chi_{\perp}$ and $\varepsilon_{z z}$ vs. asymmetry parameter $h$, temperature $T$ and electron concentration $n$ are obtained. The function 


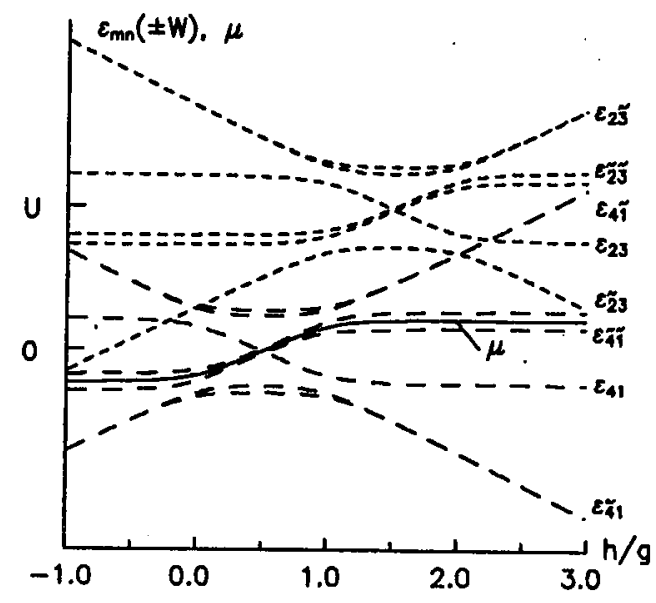

Fig. 1. The single-electron bands and chemical potential $\mu$ as functions of the dimensionless coupling strength $h / g(U=2.2, g=1, \Omega=0.3, W=0.2, n=0.7)$.

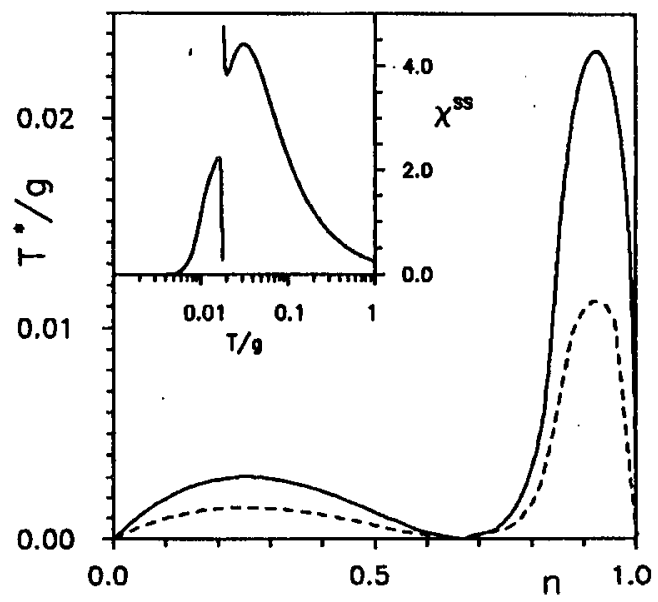

Fig. 2. Dependence of the temperature of dielectric instability $T^{*}$ on electron concentration $n(U \rightarrow \infty, \Omega=0, W=0.2, h=1.1 g$ (solid line) and $h=1.5 g$ (dashed line)); the box represents the temperature dependence of $\chi_{\perp}^{\mathrm{S}}$ (given in $d_{\mathrm{e}}^{2} / v_{c} g$ units; $h=1.1 g$, $n=0.9$ ).

$\chi_{\perp}^{\mathrm{S}}=\left\langle S^{z} S^{z}\right\rangle_{\boldsymbol{q}, \omega}^{c}$ in static limit for $U \rightarrow \infty, 0 \leq n \leq 1$ has two peaks (near $h=0$ and $g$ ) caused by the approaching of each other certain hole subbands (see Fig. $1)$. The first peak disappears at $n \rightarrow 1$ and the second one vanishes at $n \rightarrow 0$. The same structure keeps for $\Omega=0, T \neq 0$. Maxima of $\left.\chi_{\perp}^{\mathrm{S}}\right|_{\omega=0}$ correspond to the points of possible dielectric instabilities.

For the regime $\mu=$ const the substantial increase in dielectric susceptibility due to the electron component $\left(\approx\langle n n\rangle^{c}\right)$ is observed when the chemical potential is within the permitted energy subband. One can obtain the estimates 
$\chi_{\perp}^{\mathrm{e}} \approx d_{\mathrm{e}}^{2} /\left(v_{c} \tilde{W}\right)$, where $\tilde{W}=\left(A_{m n}^{\sigma}\right)^{2} W\left\langle X^{m m}+X^{n n}\right\rangle$ is the subband width renormalized due to narrowing effect, and $\chi_{\perp}^{\mathrm{e}} / \chi_{\perp}^{\mathrm{S}} \approx\left(d_{\mathrm{e}}^{2} / d_{\mathrm{S}}^{2}\right)(\Omega / \tilde{W})$. By the order of magnitude $d_{\mathrm{S}} \approx Z\left(\mathrm{O}_{A}\right) \delta, d_{\mathrm{e}} \approx e D$, where $Z\left(\mathrm{O}_{A}\right)$ is the effective charge of $\mathrm{O}_{\mathrm{IV}}$ oxygen, $\delta$ is the distance between the double-well minima, and $D$ is the effective length corresponding to the transfer of electron charge from $\mathrm{CuO}$ chains to the layers. Because $\delta \ll D$, we have $\chi_{\perp}^{\mathrm{e}} \ll \chi_{\perp}^{\mathrm{s}}$ for $\Omega \leq \tilde{W}$. Direct numerical estimation gives $\chi_{\perp}^{\mathrm{e}} \approx 10^{1} \div 10^{2}$, which can match to the large observed $\varepsilon_{z z}$ permittivity values [3].

The behaviour of function $\chi_{\perp}^{\mathrm{S}}$ as well as total susceptibility $\chi$ is peculiar also with the change of temperature. For $h>g$ and $h<0(U \rightarrow \infty)$ the curve $\chi_{\perp}^{\mathrm{S}}(T)$ rapidly falls to zero when $T \rightarrow 0$ and smoothly decreases (showing Curie-Weiss like behaviour) at high temperatures. There is a divergence of $\chi_{\perp}^{\mathbf{S}}$ at certain temperature $T^{*}$, which is determined by the effective interaction between pseudospins. The phase diagram $T^{*}$ vs. $n$ is given in Fig. 2. The formal analogy between the presented diagram and well-known dependencies of the superconducting transition temperature $T_{c}$ on hole concentration in high-temperature superconductors testifies to the possible internal connection between these phenomena.

\section{References}

[1] K.A. Müller, Phase Transit. (special issue) (1988).

[2] V. Müller, C. Hucho, D. Maurer, Ferroelectrics 130, 45 (1992).

[3] L.R. Testardi, W.G. Moulton, H. Matias, H.K. Ng, C.M. Rey, Phys. Rev. B 37, 2324 (1988).

[4] I.V. Stasyuk, A. Shvaika, Acta Phys. Pol. A 84, 293 (1993).

[5] Yu.A. Izyumov, B.M. Letfulov, J. Phys., Condens. Matter 3, 5373 (1991). 